\title{
Localized Pulmonary Alveolar Proteinosis: Two Case Reports
}

\author{
Seon Jeong $\mathrm{Oh}^{1}$, Ji Yung Choo ${ }^{1}$, Ki Yeol Lee ${ }^{1}$, Je-Hyeong Kim², Suk Keu Yeom ${ }^{1}$ \\ ${ }^{1}$ Department of Radiology, Korea University Ansan Hospital, Ansan, Korea \\ ${ }^{2}$ Department of Pulmonology, Korea University Ansan Hospital, Ansan, Korea
}

Background: Pulmonary alveolar proteinosis (PAP) is a relatively rare disease characterized by abnormal accumulation of surfactantlike material in the alveolar spaces. The classic radiologic findings of PAP include bilateral, symmetric, diffuse ground-glass opacity (GGO) or consolidation. The most common computed tomography (CT) feature of PAP is widespread GGO with thickened interlobular septa, the so-called crazy-paving pattern, which strongly suggests the diagnosis.

Case Report: Here, we report the cases of two young male patients with unusual presentations of PAP. One patient showed localized
PAP in the left lower lobe on CT images and the other patient presented with unilateral PAP involving the right lower lung field and recurrence in the same area with the same pattern as the initial manifestation.

Conclusion: In conclusion, it is important for radiologists to be aware of potential atypical imaging findings of PAP in order to provide a correct diagnosis. Along these lines, PAP can present as a solitary nodular lesion or unilateral focal lesion, and can recur in the same pattern and location. (Balkan Med J 2014;31:257-60).

Key Words: CT, localized, pulmonary alveolar proteinosis
Pulmonary alveolar proteinosis (PAP) is a rare pulmonary disease characterized by accumulation of surfactant in alveolar and distal bronchi. Following the original description of PAP by Rosen et al. (1) in 1958, numerous reports of radiologic findings of PAP have appeared in the literature. The typical computed tomography (CT) finding of PAP is known as the diffusely distributed, bilateral, crazy-paving pattern (2). It is significant for radiologists and also pulmonologists to be aware of potential atypical imaging findings of PAP in order to provide a correct diagnosis. Here, we present two cases of PAP with unusual features exhibiting a localized and unilateral pattern on $\mathrm{CT}$ and discuss our diagnosis.

\section{CASE PRESENTATIONS}

Written informed consent was obtained from patients who participated in this cases.

\section{Case 1}

A 21-year-old male presented with a 2-week history of dyspnea, cough, and scanty whitish sputum. The patient had no specific underlying disease and denied any history of smoking, allergies, or drug abuse. After admission, physical examination revealed a crackle in the patient's left lower lung lobe. A chest CT obtained 8 days before admission at a local clinic showed a 1.2 -cm-sized solitary partly-solid nodule which consisted of both ground-glass and solid softtissue attenuation components interpreted using factors proposed by the Fleischner Society Nomenclature Committee (3) (Figure 1). Initially, we considered this lesion as focal bacterial pneumonia or eosinophilic pneumonia; however, the patient did not complain of any signs of infection, such as fever or chills. Although erythrocyte sedimentation rate (ESR) was slightly elevated at $13 \mathrm{~mm} / \mathrm{hr}$, there was no evidence of infection on the other laboratory findings with white blood cells (WBC), $8,500 \mathrm{~mm}^{3}$ or C-reactive protein (CRP), $0.3 \mathrm{mg} / \mathrm{dL}$. And the results of arterial blood gas analysis (ABGA) and spirometric tests were within normal limits. Furthermore, CT identified only a solitary lesion without eosinophilia, WBC eosinophil ratio, 2.6\%. A follow-up CT performed at the time of admission demonstrated consolidation with peripheral GGO and interlobular septal thickening, demonstrating a crazy-paving pattern in the left lower lobe, the extent of which was increased compared with the initial CT scan (Figure 2). In addition, laboratory findings revealed no significant abnormality in the patient's peripheral blood or sputum. We then performed a video-associated thoracoscopic (VATS) biopsy to obtain a diagnosis. Pathologic examination demonstrated marked filling of the alveo- 


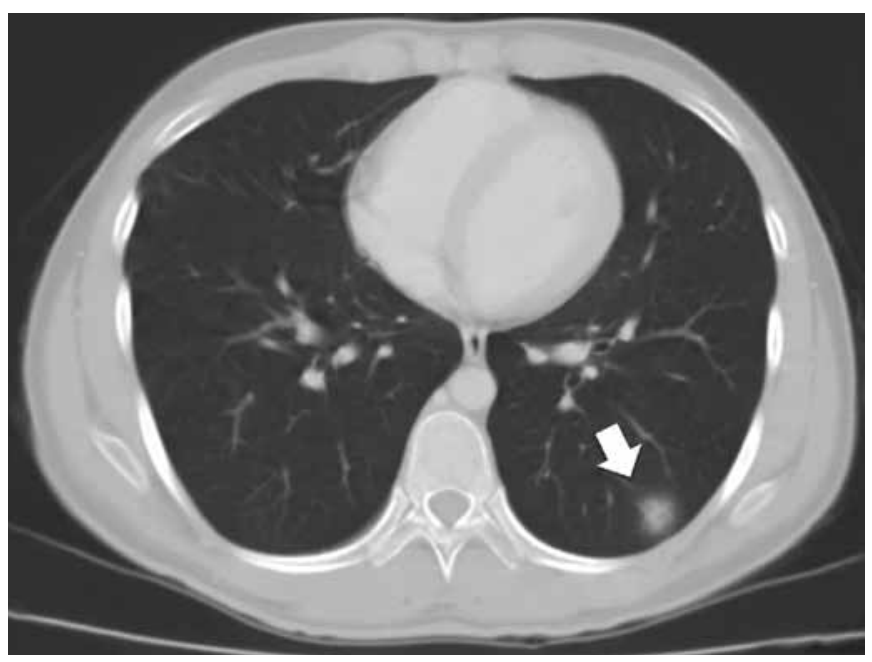

FIG. 1. Initial chest CT images of Case 1 . An approximately $2 \mathrm{~cm}$ sized subsolid nodule (arrow) with a peripheral halo was noted in the left lower lobe

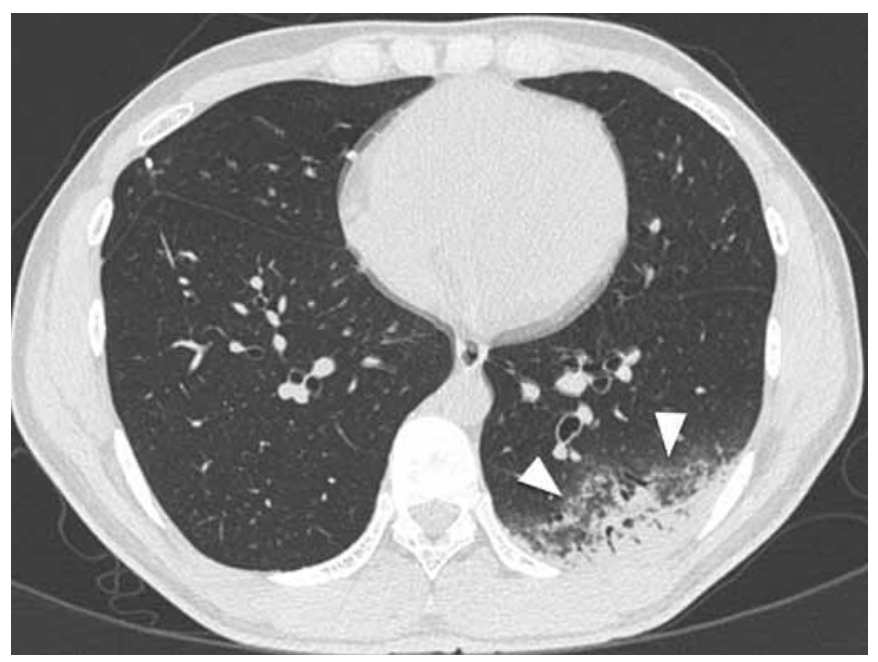

FIG. 2. Follow up CT images of Case 1. Follow up CT performed 10 days later demonstrated patchy consolidation with a crazy-paving pattern appearance (arrowhead)

lar spaces with a granular eosinophilic material that stained positive with periodic acid Schiff (PAS), and the patient was subsequently diagnosed with PAP. Clinical symptoms were improved with conservative treatment, and there was no evidence of PAP lesions present on a follow-up CT performed one year later.

\section{Case 2}

A 37-year-old male presented with cough and whitish sputum lasting for 15 days. The patient also suffered from dyspnea in the supine position for 3 days. The patient was put on empirical antibiotics by a local clinic for one week for suspicion of pneumonia, but continued to complain of cough, sputum, and dyspnea without improvement. He was diagnosed as an HBV carrier, and denied any other history of smoking, allergies, or drug

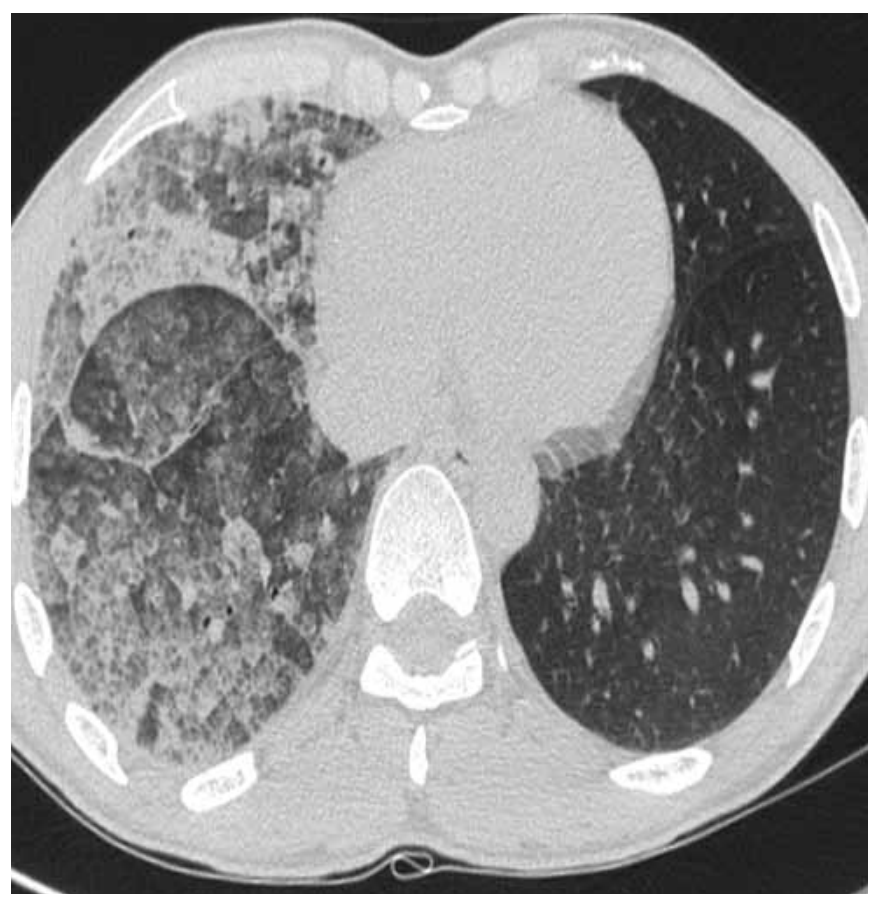

FIG. 3. Initial CT images of Case 2. Axial CT scans with a lung window setting demonstrate ground-glass opacity (GGO) with prominent interlobular septal thickening (crazy-paving pattern) only in the right middle and lower lobes

abuse. After admission, physical examination demonstrated a crackle in the right mid to lower lung lobes. Peripheral blood assay demonstrated normal results of WBC: $9,510 / \mathrm{mm}^{3}, \mathrm{Hb}$ (haemoglobin): $15.2 \mathrm{~g} / \mathrm{dL}$, and platelets: $180,000 / \mathrm{uL}$, and ESR was elevated at $44 \mathrm{~mm} / \mathrm{hr}$. The results of arterial blood gas analysis (ABGA) and spirometric tests were within normal limits. Acidfast bacilli (AFB) stains and cultures of sputum were negative.

Initial chest radiography showed diffuse GGO and consolidations in the right mid to lower lung zone. Chest CT showed GGO and consolidation with interlobular septal thickening (crazy-paving pattern) in the right middle lobe and right lower lobe (Figure 3). The patient subsequently underwent bronchoscopy, which revealed that the right middle lobar bronchus was filled with a sticky, off-white secretion without evidence of intraluminal lesions. A bronchial washing test revealed no specific findings. A VATS biopsy was then performed for confirmative diagnosis, which confirmed PAP with PAS positive granular eosinophilic material in the alveolar spaces. A follow up CT performed 4 months after operation revealed improved diffuse GGO and consolidations in the right lung (Figure 4). An HRCT scan obtained when symptoms recurred after 11 months showed widespread ground-glass opacity and interlobular septal thickening in the right middle and lower lobes in the same area where the PAP was previously noted (Figure 5). After conservative treatment, the clinical and radiological findings were improved. 


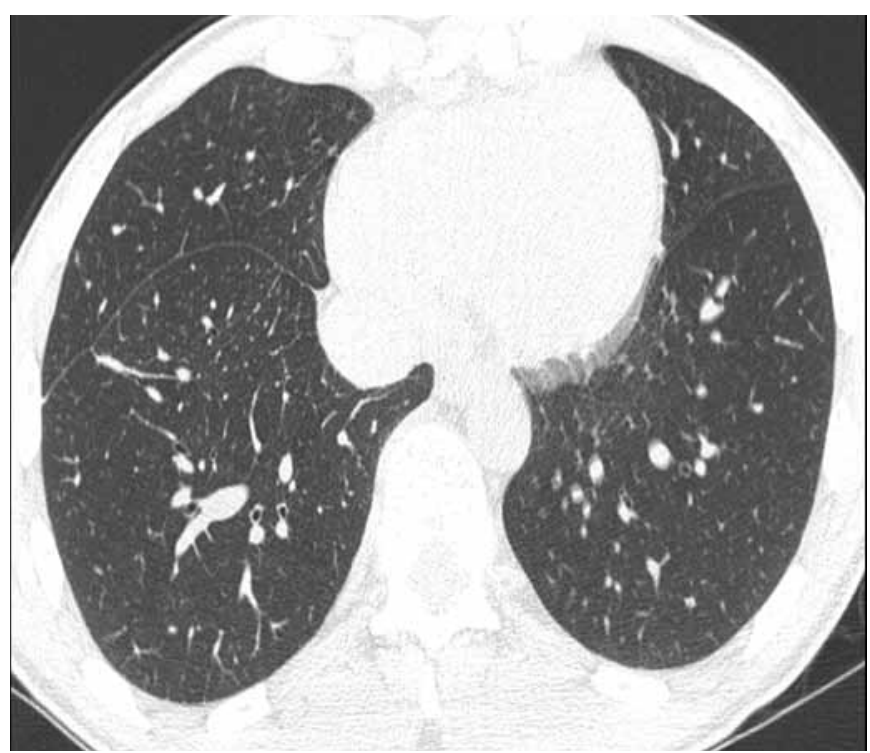

FIG. 4. Follow up CT images performed 4 months later of Case 2. Follow-up CT scan performed 4 months later demonstrating resolved GGO without definite abnormal findings

\section{DISCUSSION}

Pulmonary alveolar proteinosis is a relatively rare disease that is classified into idiopathic and secondary forms based on the presence of haematologic malignancy, compromised immunity, or exposure to materials such as silica and cement (1-4). PAP consists of intra-alveolar accumulation of lipoproteinaceous material resulting from a defective turnover of surfactant (5). In addition, there is typically a uniform accumulation of PAS-positive material in all alveolar spaces. Electron microscopy shows that the intraalveolar material consists of amorphous, granular debris containing numerous osmiophilic, fused membrane structures with a periodicity of $4.7 \mathrm{~nm}$ and resembling lamellar bodies and tubular myelin $(6,7)$

Therapy for secondary PAP generally involves treatment of the underlying condition. Acquired PAP has been treated successfully since the early 1960 s by whole-lung lavage, and this treatment remains the standard of care today. Several prospective phase 2 trials of GM-CSF therapy for acquired PAP have been undertaken. The prognosis for acquired PAP varies, but disease-specific survival rate exceeds $80 \%$ at 5 years (7).

Although CT identifies PAP as various image findings ranging from GGO to dense consolidation, typical CT features strongly suggest that PAP is GGO with interlobular septal thickening (crazy-paving appearance). Likewise, these lesions are distributed diffusely or geographically involving the bilateral lung parenchyma $(8,9)$.

Teja et al. (9) reported a case of scattered foci of PAP while Mildenberger et al. (10) reported that focal congenital PAP as-

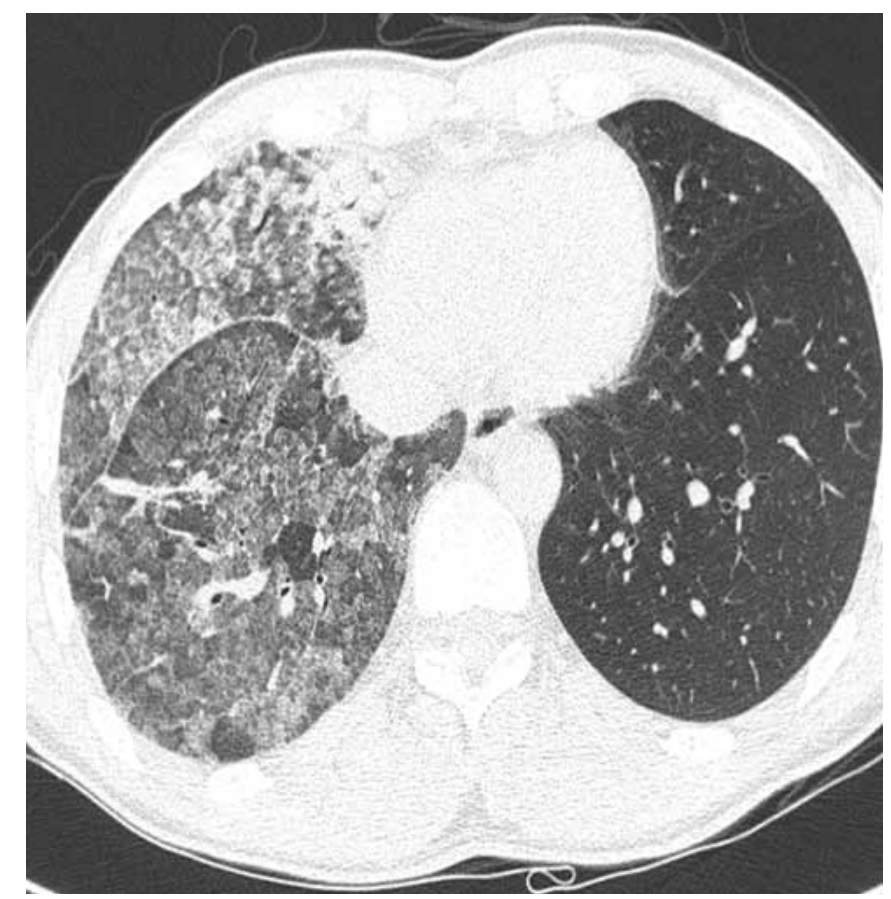

FIG. 5. Follow up CT images performed 11 months later of Case 2. HRCT scan obtained when symptoms recurred after 11 months showing recurrent widespread ground-glass opacity and interlobular septal thickening in the same area of the right middle and lower lobes

sociated with abnormal surfactant protein B messenger RNA is identified only by thoracoscopic imaging. Midenberger et al. (10) suggested that focal accumulation of PAS-positive alveolar proteinaceous material can present as focal PAP.

With respect to radiologic findings, there have been no reports of localized or unilaterally recurring patterns of PAP aside from the description by L. Boussoffara et al. (11) and Prakash et al. (12) of 6 cases of unilateral or asymmetric patterns of PAP. Indeed, the majority of the literature investigating CT findings of PAP have reported the diffusely and bilaterally distributed crazy-paving appearance. In the two cases reported here, one patient demonstrated a small nodule with an adjacent GGO halo that was initially considered as eosinophilic pneumonia or a focal inflammatory lesion. However, the nodule with a CT halo changed to a patchy consolidation; this nodular and localized pattern of PAP was considered to be a very rare manifestation. The second case demonstrated a relatively common finding as a unilateral consolidation with GGO and crazy-paving appearance, but was interesting in that recurrent PAP was found 14 months later in the same pattern and location, unilaterally. In conclusion, it is important for radiologists to be aware of potential atypical imaging findings of PAP in order to provide a correct diagnosis. Along these lines, PAP can present as a solitary nodular lesion or unilateral focal lesion, and can recur in the same pattern and location. 


\section{Ethics Committee Approval: N/A.}

Informed Consent: Written informed consent was obtained from the patients for the publication of this case report and any accompanying images.

Peer-review: Externally peer-reviewed.

Author contributions: Concept - J.Y.C., K.Y.L.; Design - J.Y.C.; Supervision - K.Y.L.; Resource - K. Y.L.; Materials - J.Y.C.; Data Collection\&/or Processing - J-H.K., S.J.H., J.Y.C.; Analysis\&/or Interpretation - J.Y.C., S.J.O.; Literature Search - J.Y.C., S.J.O.; Writing - S.J.O.; Critical Reviews - S.K.Y.

Conflict of Interest: No conflict of interest was declared by the authors.

Financial Disclosure: The authors declared that this report has received no financial support.

\section{REFERENCES}

1. Rosen SH, Castleman B, Liebow AA. Pulmonary alveolar proteinosis. $N$ Engl J Med 1958;258:1123-42. [CrossRef]

2. Frazier AA, Franks TJ, Cooke EO, Mohammed TL, Pugatch RD, Galvin JR. From the archives of the AFIP: Pulmonary alveolar proteinosis. $R a-$ diographics 2008;28:883-99. [CrossRef]
3. David MH, Alexander AB, Heber M, Theresa CM, Nestor LM, Jacques R. Fleischner Society: Glossary of terms for thoracic imaging. Radiology 2008;246:697-722. [CrossRef]

4. Presneill JJ, Nakata K, Inoue Y, Seymour JF. Pulmonary alveolar proteinosis. Clin Chest Med 2004;25:593-613. [CrossRef]

5. Singh G, Katyal SL, Bedrossian CW, Rogers RM. Pulmonary alveolar proteinosis. Staining for surfactant apoprotein in alveolar proteinosis and in conditions simulating it. Chest 1983;83:82-6. [CrossRef]

6. Bruce CT, Jeffrey AW, Koh Nakata. Pulmonary Alveolar Proteinosis. $N$ Engl J Med 2003;349:2527-39. [CrossRef]

7. Dirksen U, Nishinakamura R, Groneck P, Hattenhorst U, Nogee L, Murray $\mathrm{R}$, et al. Human pulmonary alveolar proteinosis associated with a defect in GM-CSF/IL-3/IL-5 receptor common beta chain expression. $J$ Clin Invest 1997;100:2211-7. [CrossRef]

8. Holbert JM, Costello P, Li W, Hoffman RM, Rogers RM. CT features of pulmonary alveolar proteinosis. AJR Am J Roentgenol 2001;176:1287-94. [CrossRef]

9. Teja K, Cooper PH, Squires JE, Schnatterly PT. Pulmonary alveolar proteinosis in four siblings. $N$ Engl J Med 1981;305:1390-2. [CrossRef]

10. Mildenberger E, deMello DE, Lin Z, Kössel H, Hoehn T, Versmold HT. Focal congenital alveolar proteinosis associated with abnormal surfactant protein B messenger RNA. Chest 2001;119:645-7. [CrossRef]

11. Boussoffara L, Boudawara N, Knani J, Zantour B, Khouaja I. Protéinose alvéolaire atypique. Rev Pneumol Clin 2011;67:158-62. [CrossRef]

12. Prakash UB, Barham SS, Carpenter HA, Dines DE, Marsh HM. Pulmonary alveolar phospholipoproteinosis: Experience with 34 cases and a review. Mayo Clin Proc 1987;62:499-518. [CrossRef] 